\title{
Characteristic Analysis from Excessive to Deficient Syndromes in Hepatocarcinoma Underlying miRNA Array Data
}

\author{
Qi-Long Chen, ${ }^{1,2}$ Yi-Yu Lu, ${ }^{1}$ Gui-Biao Zhang, ${ }^{1}$ Ya-Nan Song,, Qian-Mei Zhou, ${ }^{1}$ Hui Zhang, \\ Wei Zhang, ${ }^{3}$ Xin-sheng Tang, ${ }^{2}$ and Shi-Bing Su${ }^{1}$ \\ ${ }^{1}$ Research Center for TCM Complexity System, Shanghai University of TCM, Shanghai 201203, China \\ ${ }^{2}$ College of Life and Environment Science, Huangshan University, Huangshan 245021, China \\ ${ }^{3}$ Shanghai Longhua Hospital, Shanghai University of TCM, Shanghai 200126, China \\ Correspondence should be addressed to Shi-Bing Su; shibingsu07@163.com
}

Received 18 August 2013; Accepted 12 November 2013

Academic Editor: Shao Li

Copyright (C) 2013 Qi-Long Chen et al. This is an open access article distributed under the Creative Commons Attribution License, which permits unrestricted use, distribution, and reproduction in any medium, provided the original work is properly cited.

\begin{abstract}
Traditional Chinese medicine (TCM) treatment is regarded as a safe and effective method for many diseases. In this study, the characteristics among excessive, excessive-deficient, and deficient syndromes of Hepatocellular carcinoma (HCC) were studied using miRNA array data. We first calculated the differentially expressed miRNAs based on random module $t$-test and classified three TCM syndromes of HCC using SVM method. Then, the weighted miRNA-target networks were constructed for different TCM syndromes using predicted miRNA targets. Subsequently, the prioritized target genes of upexpression network of TCM syndromes were analyzed using DAVID online analysis. The results showed that there are distinctly different hierarchical cluster and network structure of TCM syndromes in HCC, but the excessive-deficient combination syndrome is extrinsically close to deficient syndrome. GO and pathway analysis revealed that the molecular mechanisms of excessive-deficient and deficient syndromes of HCC are more complex than excessive syndrome. Furthermore, although excessive-deficient and deficient syndromes have similar complex mechanisms, excessive-deficient syndrome is more involved than deficient syndrome in development of cancer process. This study suggested that miRNAs might be important mediators involved in the changing process from excessive to deficient syndromes and could be potential molecular markers for the diagnosis of TCM syndromes in HCC.
\end{abstract}

\section{Introduction}

Hepatocellular carcinoma (HCC) is one of the most common devastating cancer types, ranking sixth in incidence, and the third leading cause of cancer related deaths worldwide $[1,2]$. The hepatitis B and hepatitis C infections, idiopathic cirrhosis, and alcoholic liver diseases usually are major risk factors for HCC [3]. For early diagnosis of HCC is difficult, and more than half of the HCC patients are diagnosed too late to benefit from the curative therapies. Furthermore, molecular approaches have revealed many molecular events associated with HCC, yet molecular mechanism of cell proliferation and effective biological markers for driving hepatocarcinogenesis remain largely unknown [4].

Under these circumstances, many failing patients seek to get help from complementary and alternative medicine, especially traditional Chinese medicine (TCM). TCM is an ancient Chinese medicine that evolved through at least three thousand years of uninterrupted clinical practice. The TCM treatment usually uses a traditional diagnosis method to classify the TCM syndromes, which based on clinical symptoms and signs, followed by the use of individualized treatment $[5,6]$. For instance, Li et al. established a network balance model to evaluate the imbalanced network underlying TCM syndrome for gastritis patients $[7,8]$, which demonstrated that cold syndrome patients experience low levels of energy metabolism and immune regulation is intensified in hot syndrome patients.

MicroRNAs (miRNAs) are endogenous, noncoding, single-stranded small RNA molecules of approximately 22 nucleotides, which function as negative regulators that involve posttranscriptional gene expression through binding 
to the $3^{\prime}$-untranslated regions ( $3^{\prime}$-UTRs) of target mRNAs and consequently lead to mRNA cleavage or translational repression [9-11]. Although miRNAs are stable in circulation systems, tissue, and organ [12], they often can be explored in blood under pathological conditions, such as cell turnover and destruction, and pathological injury [13]. In the previous studies, we reported that circulating miR-583 and miR-663 refer to TCM syndrome differentiation in chronic hepatitis $B$ [14] and the progression from excessive to deficient syndromes in chronic hepatitis B using miRNA-target dynamical network [15]. The results implicated that miRNAs are important mediators for TCM syndrome classification as well as $\mathrm{CHB}$ development progression and therefore could be potential diagnosis and therapeutic molecular markers.

In this work, we hypothesized that miRNAs expression levels and their target gene expression networks are the important factors for the TCM syndrome classification and the changes from excessive to deficient syndromes in HCC patients. We thus focused on the comparative analysis of the differences and similarities in the three TCM syndromes including liver-gallbladder dampness heat syndrome (LGDHS), liver depression and spleen deficiency syndrome (LDSDS), and liver-kidney yin deficiency syndrome (LKYDS). The aim is to demonstrate the change process of molecular mechanism from excessive syndrome to deficient syndrome at a network level by an integrative and comparative analysis of weighted miRNA-target network in HCC patients.

\section{Materials and Methods}

2.1. Clinical Specimens. In this work, clinical serum of 9 HCC patients and 7 healthy donors (Normal) were collected, whose were come from Shanghai Longhua Hospital. Then, these serums were subjected to miRNA microarray analysis. The diagnostic criteria of western medicine for HCC followed the guidelines defined by the Chinese Society of Hepatology and Chinese Society of Infectious Diseases in 2005 [16]. The TCM syndrome system for HCC applied by the 3 senior TCM doctors of each diagnosis was accepted according to the standards of TCM differential syndromes of viral hepatitis defined by the Internal Medicine Hepatopathy Committee of Chinese Traditional Medicine Association in 1991 [17]. This research project was approved by the local ethics committee of Shanghai University of TCM, and all patients were informed and consented for this study.

The differentiation of TCM syndromes in HCC patients was shown in Table 1. There were 3 LGDHSs, 3 LDSDSs, and 3 LKYDSs. In addition, 7 serums of normal control were randomly obtained from 120 individuals who had physically examination at Shanghai Longhua Hospital.

2.2. Serum Sample Collection and RNA Isolation. All serum samples were from the peripheral venous blood of HCC patients and healthy donors, which were immediately frozen in liquid nitrogen and then stored at $-80^{\circ} \mathrm{C}$. The RNAs in serum were extracted using a miRVana PARIS kit (Ambion, Austin, TX, USA) according to the manufacturer's protocol
TABLE 1: Differentiation of TCM syndromes in HCC patients.

\begin{tabular}{lcccc}
\hline $\begin{array}{l}\text { Patient } \\
\text { ID }\end{array}$ & Age & Gender & $\begin{array}{c}\text { TCM } \\
\text { syndromes }\end{array}$ & $\begin{array}{c}\text { TCM } \\
\text { syndrome types }\end{array}$ \\
\hline HCC 1 & 47 & M & LGDHS & Excessive \\
HCC 2 & 63 & M & LGDHS & Excessive \\
HCC 3 & 61 & F & LGDHS & Excessive \\
HCC 4 & 62 & M & LDSDS & Excessive-deficient \\
HCC 5 & 59 & M & LDSDS & Excessive-deficient \\
HCC 6 & 58 & M & LDSDS & Excessive-deficient \\
HCC 7 & 54 & F & LKYDS & Deficient \\
HCC 8 & 52 & M & LKYDS & Deficient \\
HCC 9 & 42 & M & LKYDS & Deficient \\
\hline
\end{tabular}

and using the RNase-free DNase (Promega, Madison, WI, USA) to eliminate DNA contamination. The concentration of RNAs isolated from serum ranged from 1.5 to $12 \mathrm{ng} / \mu \mathrm{L}$.

2.3. miRNA Microarray and Data Analysis. The profiles of serum miRNAs of 9 HCC patients and 7 normal controls were generated using Agilent Human miRNA microarray V3 (Agilent Technologies Inc, Santa Clara, CA, USA); 60 ng of RNA was labeled and hybridized for each array. Hybridization signals were detected with the Agilent microarray scanner; the data were extracted using Feature Extraction V10.7 (Agilent Technologies, CA, USA). All raw data were transformed into $\log 2$ scale, and then, the expression levels were normalized by having zero mean and unit sample variance.

In order to evaluate the diversity of three TCM syndromes in HCC, we compared the miRNAs expressions of LGDHS, LDSDS, and LKYDS to normal, respectively. The relative miRNA expression levels were further normalized utilizing the median over the all patients, which make the each patient have a median log ration of 0 in normalized expression levels. The weighted differences miRNAs between TCM syndromes were calculated using the random variance model $t$-test and the fold-change $>1.5$ and $P<0.05$ were considered significant. Heat-map analysis and hierarchical cluster analysis of expression data were performed using Cluster 3.0 and TreeView programs (the clustering calculation uses one minus correlation metrics and average linkages). Class prediction was performled using a statistical algorithm of the support vector machine (SVM) incorporating miRNA differentially expressed at a univariate parametric significance level of $P=$ 0.01 . The prediction rate was estimated via 10 fold and 10 times cross-validation and the bootstrap method for small sample data.

2.4. Identification and Prediction of miRNA Target Genes. Validated miRNA target genes were selected based on TarBase 6.0, which hosts the largest collection of manually curate experimentally validated miRNA-gene interactions [18]. Furthermore, the unverified miRNA target genes were predicted to regulating by miRNAs based on 10 programs, including DIANAmT, miRanda, miRDB, miRWalk, RNAhybrid, PicTar4, PicTar5, PITA, RNA22, and TargetScan. In 
these programs, the miRDB is different from others, which was using SVM learning machine to predict miRNA targets [19]. In order to increase the accuracy of predicted targets, we further screened prediction hits from two ways, that is, (i) random selected two sets from 9 programs (excepted miRDB) and intersected them, respectively, then united these intersection data as Data A; (ii) selected the miRDB data whose score $>60$, defined this data is Data B. Finally, the intersection between Data A and Data B acts as final data to build miRNA-target network.

2.5. Enrichment Analysis of Target Genes. Of the inferred miRNA target genes, those showing a significant $(P<0.05)$ expression difference between normal, LGDHS, LDSDS, and LKYDS samples were analyzed for pathways involving these genes using DAVID online analysis [20, 21], and significance analysis was determined when $P$ values were corrected for false discovery rate (FDR). Gene sets containing less than 5 genes overlapping were removed from the DAVID analysis. In our analysis, GO terms and pathways with an FDR-adjusted $P$ value of less than 0.05 were retained.

2.6. Weighted miRNA-Target Network Construction. We built the weighted miRNA-target gene networks for different syndromes of HCC by computing the miRNA and target gene degree distribution based on experimental validated target genes and predicted target genes, thus inferring the miRNAtarget network in 3 TCM syndromes of HCC, respectively. In the process of network building, miRNA nodes were weighted by their expression fold changes (absolute value of $\log 2$ ), while target genes were weighted based on degree distributions between consecutive groups, and thus we obtained a node-weighted miRNA-target interaction network for each stage. In order to validate the veracity of above network, rank all nodes (miRNAs and target genes) of network according to their weights and test the similarity between them [15, 22]; thereafter, obtain deregulated nodes for mapping the network of consecutive TCM syndromes progression. In the weighted miRNA-target network, the nodes represent miRNAs or genes, and the edges represent the connection strength (adjacency).

\section{Results and Discussion}

3.1. Differential Expressed miRNAs of TCM Syndromes in HCC. The expressions of miRNAs were calculated and analyzed with random module $t$-test of $\mathrm{R}$ package, to search whether there are some significantly differential expressed miRNAs among the consecutive stages form excessive syndromes to deficient syndromes. 35 miRNAs in LGDHS/normal, 61 miRNAs in LDSDS/normal, and 71 miRNAs in LKYDS/normal differentially expressed. Three TCM syndromes of HCC were classified using a supervised learning algorithm (binary tree classification), and SVM act as prediction method. As shown in Figure 1(a), the excessive syndrome (LGDHS) was clearly classified to excessivedeficient combination syndrome (LDSDS) and deficient syndrome $($ LKYDS) (node 1, score $=82$ ), which implicated that the excessive syndrome was distinctly different for other syndromes in HCC patients. However, we notice that the LDSDS samples first classified two classifications (node 2, score $=86$ ) and then form a parallel branch cluster to LKYDS (node 3, score $=63$ ). Although the topological profiles between LDSDS and LKYDS is clear-cut (Figure 1(a)), the lower SVM score (node 3, score $=63$ ) shows the classification of them is still weakly. This result suggests the relationship between Excessive-deficient combination syndrome and Deficient syndrome is extrinsically closely in HCC patients.

Hierarchical cluster analysis revealed that the expression profiles of the differential miRNAs from each TCM syndrome were roughly classified, respectively. The consecutive heatmaps of differentially expressed miRNAs were shown in Figure 1(b). With the heat-maps, the miRNAs expression profiles of three syndromes were great differences, especially the Excessive syndrome (LGDHS/Normal) has great diversity to other syndromes. It implicates that the mechanism is individual for different typical syndromes of HCC. Interestingly, the hierarchical analysis shows that three LDSDS samples and one normal control (N3) first form a parallel branch and then cluster to other normal samples. This characteristic also was corresponding with the above profiles of LDSDS classification (Figure 1(a)). Because excessive-deficient combination syndrome (LDSDS) is a complicated TCM syndrome that includes both excessive syndrome and deficient syndrome [15], we infer that the LDSDS syndrome might have two features, which is compatible for excessive syndrome and deficient syndrome, and the miRNA-regulated mechanism is more complex than other syndromes in HCC.

3.2. Overview of the miRNA-Target Networks and Network Connections. As a gene regulator, a given miRNA usually has multiple different mRNA targets, and multiple miRNAs might target one gene [23]. In this study, using the validation database (Tarbase 6.0) and 10 predicted programs, miRNA target genes of three TCM syndromes were predicted, respectively. The final predictions were obtained by significant differences $(P<0.05)$ in each prediction program. Following the differential expressed miRNAs among LGDHS, LDSDS, and LKYDS in HCC, miRNA-target network for each syndrome was reconstructed based on predicted data. The global profiles of networks were shown in Figure 2(a). Noticeably, the topological profiles are more likely closed to "medusa" architecture [24], which consists of a regulatory core of hub nodes represented most prominently by miRNAs and target genes in network. It implies that the hub nodes (miRNAs or target genes) are much stronger determinants of the realized gene expression profiles, whereas the periphery nodes that should be regulated are not regulating. Furthermore, it also implicates the potential modules are subsistent in the networks, which in biological networks often represent molecular complexes and pathways [22].

To reveal the details of the regulatory core of network, the simplified network was reconstructed through the selected hub nodes. In this work, we defined a hub node that has more than 5 interactions in those TCM syndromespecific networks, which represent these hub nodes might 


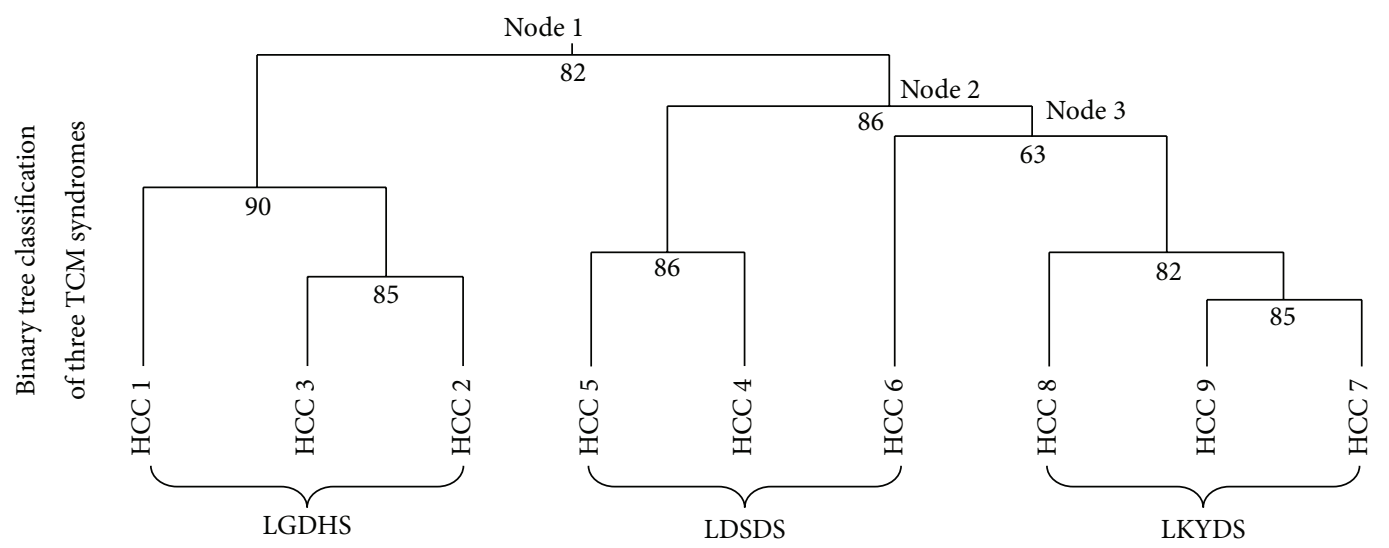

(a)

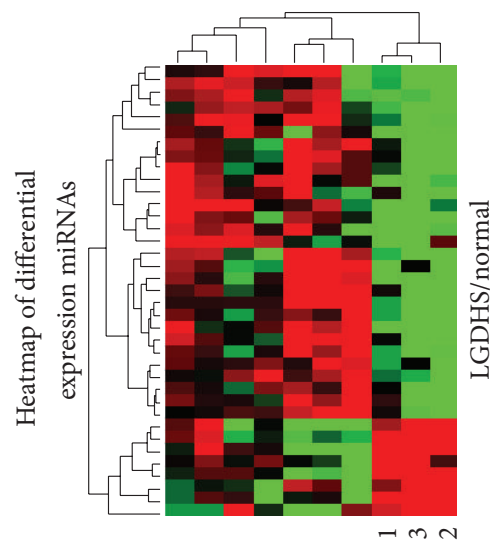

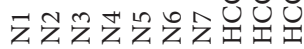

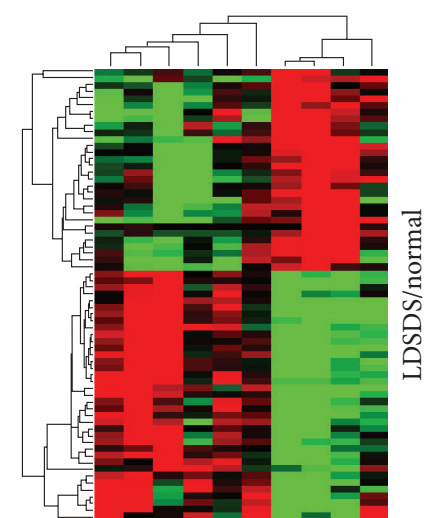

ชூ

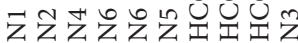

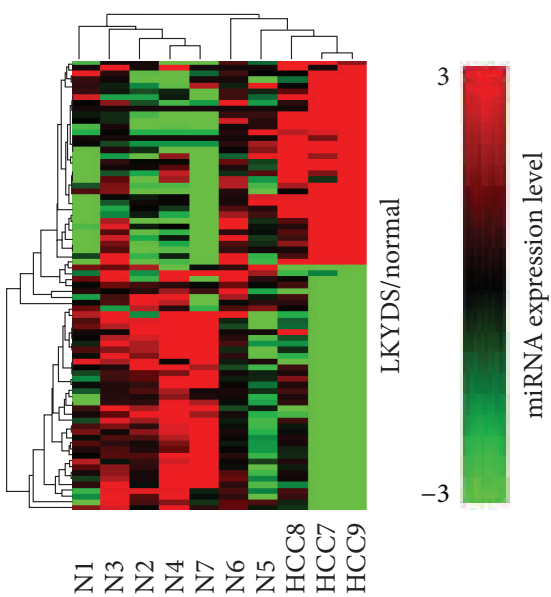

(b)

FIGURE 1: Cluster analysis of differential expression miRNAs in TCM syndromes in HCC. (a) Relationship among three typical syndromes of HCC divided by binary tree classification. (b) Heatmap of differential expressed miRNAs among the LGDHS/normal, LDSDS/normal, and LKYDS/normal.

have highly effects for the topological structure of network. The reconstructed networks were shown in Figure 2(b), including the excessive syndrome (LGDHS/normal) network that consists of 35 miRNAs, excessive-deficient syndrome (LDSDS/normal) network that consists of $61 \mathrm{miRNAs}$, and Deficient syndromes (LKYDS/normal) network that consists of 71 miRNAs. Furthermore, there were 22 coexpression miRNAs between LGDHS/normal and LDSDS/normal, including 6 upexpression miRNAs. $41 \mathrm{miRNAs}$ overlapped between LDSDS/normal and LKYDS/normal, and 5 miRNAs were upexpression; subsequently, LGDHS/normal shared 22 miRNAs with LKYDS/normal network, and including 12 upexpression miRNAs. The expression levels of overlapping miRNAs were shown in Figure 2(c). Although these overlapping miRNAs were appearance in three TCM syndromes, the difference of expression levels still indicated that they might play different roles form excessive to deficient syndromes in HCC. On the other side, the poor overlapping upexpression miRNAs also show a dramatic difference of deregulation in TCM syndromes, which suggested that LGDHS, LDSDS, and LKYDS have different molecular mechanisms.
3.3. Networks Prioritized Target Genes and Pathways in TCM Syndromes Progression in HCC. Because upexpression miRNAs might play roles that are more important in biological process, we divided the up-expression miRNA-target networks from the holistic networks for each TCM syndrome. Figure 3(a) shows the up-expression networks (degree $\geq 5$ ). We note that the proportion of up-expression miRNAs in LGDHS/normal is $18.8 \%$, which was stringently less than LDSDS/normal (37.0\%) and LKYDS/normal (39.7\%). This great difference suggests that the molecular mechanism of excessive-deficient syndrome and deficient syndromes is more complex than excessive syndrome for HHC patients. Actually, these hub up-expression miRNAs usually affected the core cellular functions, such as immune responses and cell cycle in the molecular network via inhabited or degraded target genes [15]; furthermore, it also provides a new approach to distinguish the functional processes in disease progression $[25,26]$.

Because miRNA general inhibit translation or induce mRNA degradation by binding to the $3^{\prime}$-UTRs of target mRNAs [11]; here, we focus to conduct the GO terms and 


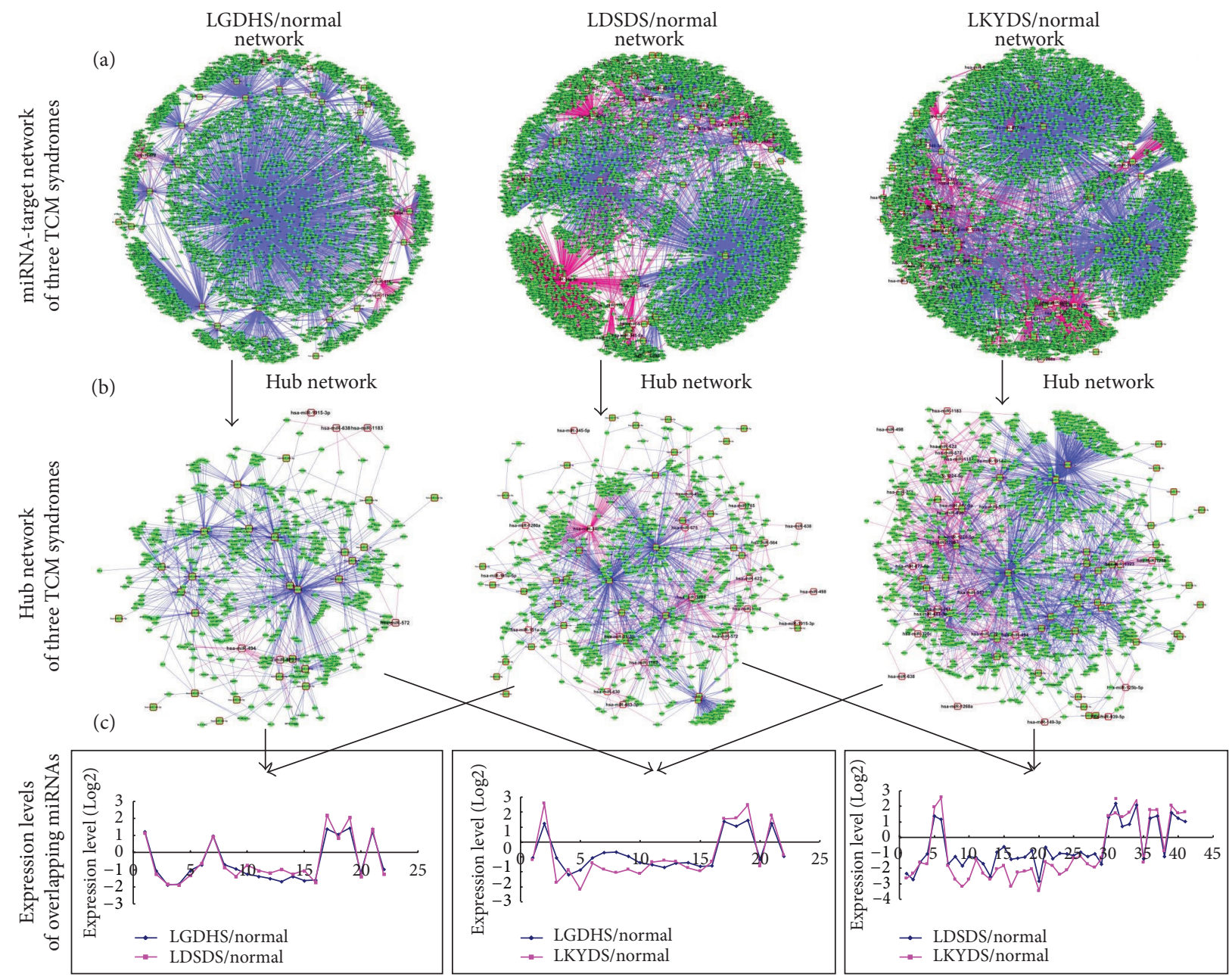

FIGURE 2: The miRNA-target networks and overlapping miRNA expression in TCM syndromes in HCC. (a) The global profiles of miRNAtarget networks in three HCC TCM syndromes. (b) The hub networks of three syndromes from excessive to deficient syndromes. (c) The overlapping miRNA expression levels from the networks of three TCM syndromes.

pathways analysis for the target genes of up-expression miRNAs using DAVID analysis [20,21]. Significant analysis was determined when $P$ values were corrected for false discovery rate (FDR). Gene sets containing greater than 5 genes overlapping were retained from the DAVID analysis. Analysis results with an FDR-adjusted $P$ value of less than 0.05 were retained.

The representative GO and pathways terms of target genes in each TCM syndrome were shown in Figure 3(b). With the GO analysis, 21 GO terms were overlapping between LGDHS/normal and LDSDS/normal; 18 GO terms are overlapping between LGDHS/normal and LKYDS/normal, while LDSDS/normal and LKYDS/normal have 107 GO terms in common. In order to understand the GO terms holistically, 18 overlapped GO terms among LGDHS, LDSDS, and LKYDS were depth selected from original results based on $P$ value and FDR less than 0.05. The distribution of overlapping GO terms was shown in Figure 3(c). Although these overlapping GO terms were appearance, actually, the FDR-adjusted $P$ value of each GO term is different. For instance, the $P$ values of GO: 0003677 (DNA binding), GO: 0031981 (nuclear lumen), GO: 0044451 (nucleoplasm part), GO: 0048522 (positive regulation of cellular process) and GO: 0070013 (intracellular organelle lumen) in LKYDS are clearly less than LGDHS or LDSDS. These results implicated that these GO terms are more stringently associated with deficient syndrome (LKYDS) and could deregulate the core cellular functions.

The pathway terms (KEGG and BIOCARTA) of target genes were also calculated using DAVID analysis. As shown in Figure 3(b), LKYDS/normal has 15 pathways, LGDHS/normal has 13 pathways, and LGDHS/normal has only 1 pathway. In addition, LGDHS/normal and LDSDS/ normal, LGDHS/normal and LKYDS/normal only have one overlapped pathway, respectively, but LDSDS/normal and LKYDS/normal had 7 overlapped pathways. Obviously, this phenomenon suggested the mechanism of Excessive syndrome is different to other syndromes in HCC patients.

The detail of each pathway of three TCM syndromes was represented in Table 2. Compared with KEGG pathway, 


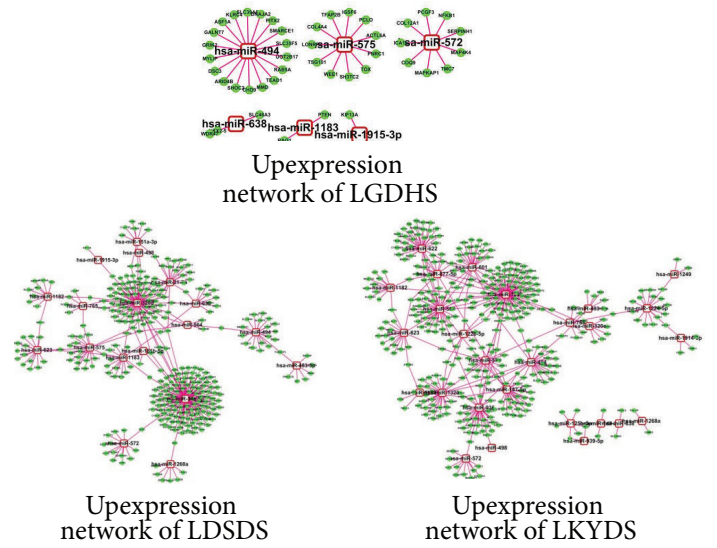

(a)

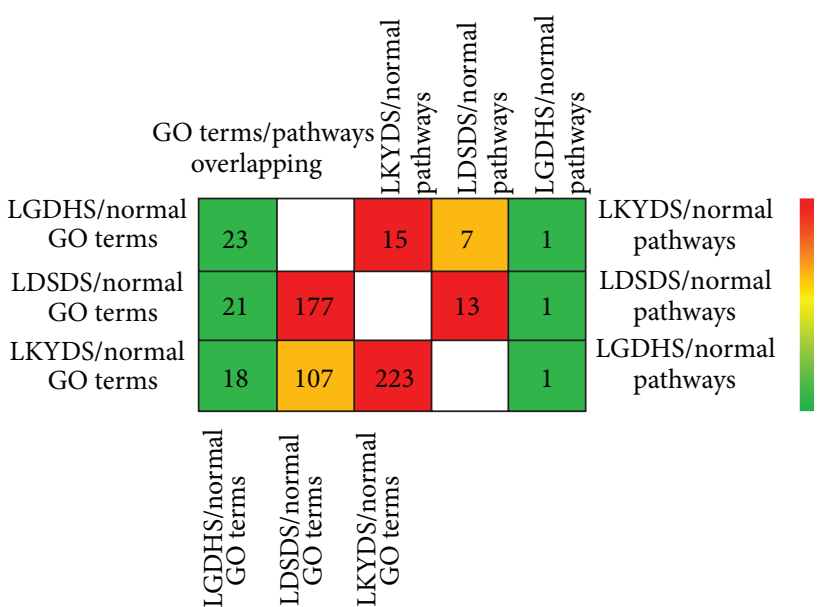

(b)

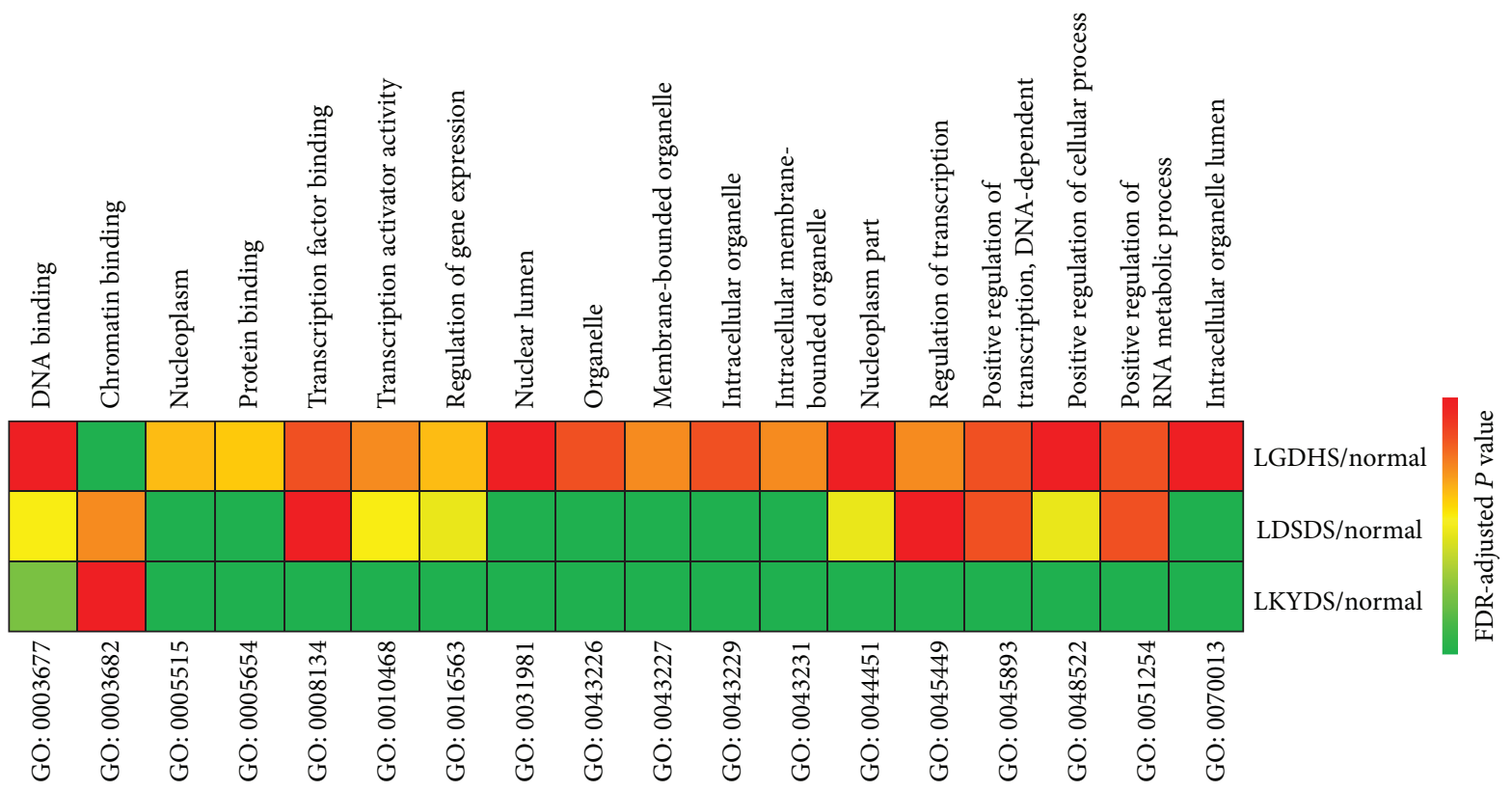

(c)

FIGURE 3: Upexpression miRNA-target networks, GO, and pathway terms in TCM syndromes progression in HCC. (a) Upexpression miRNAtarget networks of LGDHS, LDSDS, and LKYDS in HCC. (b) Comparison of GO/pathway terms among the three networks of TCM syndromes. (c) The distribution of overlapping GO terms among the three networks of TCM syndromes. Numbers in the cell represent the number of overlapped GO terms in two networks. Colors were scaled according to the proportion of overlaps.

LGDHS is only related to small cell lung cancer, LDSDS is mainly associated with cell cycle, focal adhesion, endocytosis, and cancer pathways, and apoptosis, chronic/acute myeloid leukemia, and many cancer related pathways are associated with LKYDS. Compared with BIOCARTA pathway, the Deficient syndrome (LKYDS) is mainly associated with transcriptional regulation and map kinase pathways, while excessive-deficient syndrome (LDSDS) was more likely related to cell cycle (G1/S check point), cyclins, and cell cycle regulation. Previous researches have supported that imbalance of G1/S and G2/M phases is associated with dysfunction in hepatocarcinoma [27]. The results implicated that although excessive-deficient and deficient syndromes have similar complex mechanism, excessive-deficient syndrome have more dangers than deficient syndrome involved in the development of cancer process.

\section{Conclusion}

In this study, based on miRNA microarray date of HCC patients and normal controls, we obtained evidence that the miRNA expression profiles of LGDHS, LDSDS and LKYDS are different. The classification results show that the Deficient syndrome (LKYDS) first cluster to Excessivedeficient syndrome (LDSDS), then as a parallel branch cluster to Excessive syndrome (LGDHS). Furthermore, the lower 
TABLE 2: KEGG and BIOCARTA terms distribution of downregulated genes (upexpression level of miRNAs) of TCM syndromes in HCC.

\begin{tabular}{|c|c|c|c|}
\hline Category & Term & $P$ value & FDR value \\
\hline \multicolumn{4}{|l|}{ LGDHS } \\
\hline KEGG & Small cell lung cancer & 0.0159 & 0.0141 \\
\hline \multicolumn{4}{|l|}{ LDSDS } \\
\hline KEGG & Pathways in cancer & 0.0054 & 0.0022 \\
\hline KEGG & Pancreatic cancer & 0.0142 & 0.0043 \\
\hline KEGG & Glioma & 0.0015 & 0.0157 \\
\hline KEGG & Prostate cancer & 0.0036 & 0.0171 \\
\hline KEGG & Small cell lung cancer & 0.0013 & 0.0313 \\
\hline KEGG & Non-small cell lung cancer & 0.0224 & 0.0113 \\
\hline KEGG & Cell cycle & 0.0017 & 0.0156 \\
\hline KEGG & Endocytosis & 0.0110 & 0.0233 \\
\hline KEGG & Focal adhesion & 0.0476 & 0.0436 \\
\hline BIOCARTA & Influence of Ras and Rho proteins on G1 to $\mathrm{S}$ transition & 0.0287 & 0.0144 \\
\hline BIOCARTA & Regulation of BAD phosphorylation & 0.0131 & 0.0291 \\
\hline BIOCARTA & Cyclins and cell cycle regulation & 0.0321 & 0.0323 \\
\hline BIOCARTA & Cell cycle:G1/S check point & 0.0471 & 0.0437 \\
\hline \multicolumn{4}{|l|}{ LKYDS } \\
\hline KEGG & Prostate cancer & 0.0048 & 0.0001 \\
\hline KEGG & Pathways in cancer & 0.0001 & 0.0005 \\
\hline KEGG & Glioma & 0.0001 & 0.0011 \\
\hline KEGG & Small cell lung cancer & 0.0015 & 0.0013 \\
\hline KEGG & Melanoma & 0.0019 & 0.0025 \\
\hline KEGG & Non-small-cell lung cancer & 0.0020 & 0.0021 \\
\hline KEGG & Pancreatic cancer & 0.0021 & 0.0022 \\
\hline KEGG & Chronic myeloid leukemia & 0.0072 & 0.0813 \\
\hline KEGG & Apoptosis & 0.0145 & 0.0163 \\
\hline KEGG & Acute myeloid leukemia & 0.0436 & 0.0413 \\
\hline BIOCARTA & Y branching of actin filaments & 0.0066 & 0.0771 \\
\hline BIOCARTA & NFAT and hypertrophy of the heart (transcription in the broken heart) & 0.0108 & 0.0122 \\
\hline BIOCARTA & Influence of Ras and Rho proteins on $\mathrm{G} 1$ to $\mathrm{S}$ transition & 0.0108 & 0.0122 \\
\hline BIOCARTA & Human cytomegalovirus and map kinase pathways & 0.0136 & 0.0151 \\
\hline BIOCARTA & Overview of telomerase RNA component gene hTerc transcriptional regulation & 0.0257 & 0.0268 \\
\hline
\end{tabular}

SVM score between LDSDS and LKYDS also suggested that the excessive-deficient syndrome is extrinsically close to deficient syndrome in HCC patients. The topological structure showed that the hub nodes (miRNAs or target genes) of miRNA-target network are much stronger determinants of the realized gene expression profiles, whereas the periphery nodes that should be regulated are not regulating. Obviously, the different topological profiles of networks also involved in the molecular mechanisms are different form Excessive to Deficient syndromes in HCC. GO and pathway analysis of target genes in up-expression networks revealed that the excessive-deficient and deficient syndromes of HCC are more complexity than excessive syndrome. Furthermore, although excessive-deficient and deficient syndromes have similar complex mechanism, excessive-deficient syndrome might have more dangers than deficient syndrome involved in development of cancer process.

\section{Conflict of Interests}

The authors declare that they have no financial and personal relationships with other people or organizations that can inappropriately influence their work; there is no potential conflict of interests including employment, consultancies, stock ownership, honoraria, paid expert testimony, patent applications and registrations, and grants or other funding.

\section{Acknowledgments}

This work was supported by Key Program of National Natural Science Foundation of China (813300416), National S and T Major Project of China (no. 2012ZX10005001-004), Scheming Project of Shanghai Municipal Education Commission (no. 2012JW25), Leading Project of Integrated Traditional and Western Medicine of Shanghai University of Traditional 
Chinese Medicine (2013), and Key Science Foundation of Anhui Province (KJ2012A260).

\section{References}

[1] M.-F. Yuen, J.-L. Hou, and A. Chutaputti, "Hepatocellular carcinoma in the Asia pacific region," Journal of Gastroenterology and Hepatology, vol. 24, no. 3, pp. 346-353, 2009.

[2] G.-S. Feng, "Conflicting roles of molecules in hepatocarcinogenesis: paradigm or paradox," Cancer Cell, vol. 21, no. 2, pp. 150-154, 2012.

[3] H. B. El-Serag, J. A. Davila, N. J. Petersen, and K. A. McGlynn, "The continuing increase in the incidence of hepatocellular carcinoma in the United States: an update," Annals of Internal Medicine, vol. 139, no. 10, pp. 817-823, 2003.

[4] H.-C. Hsu, Y.-M. Jeng, T.-L. Mao, J.-S. Chu, P.-L. Lai, and S.-Y. Peng, " $\beta$-catenin mutations are associated with a subset of low-stage hepatocellular carcinoma negative for hepatitis B virus and with favorable prognosis," The American Journal of Pathology, vol. 157, no. 3, pp. 763-770, 2000.

[5] W. Jia, W.-Y. Gao, Y.-Q. Yan et al., "The rediscovery of ancient Chinese herbal formulas," Phytotherapy Research, vol. 18, no. 8, pp. 681-686, 2004.

[6] Y. N. Song, J. J. Sun, Y. Y. Lu et al., "Therapeutic efficacy of fuzheng-huayu tablet based traditional chinese medicine syndrome differentiation on hepatitis-B-caused cirrhosis: a multicenter double-blind randomized controlled trail," Evidence Based Complementary Alternative Medicine, vol. 2013, Article ID 709305, 8 pages, 2013.

[7] R. Li, T. Ma, J. Gu et al., "Imbalanced network biomarkers for traditional Chinese medicine Syndrome in gastritis patients," Scientific Reports, vol. 3, article 1543, 2013.

[8] B. Jiang, X. Liang, Y. Chen et al., "Integrating next-generation sequencing and traditional tongue diagnosis to determine tongue coating microbiome," Scientific Reports, vol. 2, article 936, 2012.

[9] A. Esquela-Kerscher and F. J. Slack, "Oncomirs-MicroRNAs with a role in cancer," Nature Reviews Cancer, vol. 6, no. 4, pp. 259-269, 2006.

[10] D. P. Bartel, "MicroRNAs: target recognition and regulatory functions," Cell, vol. 136, no. 2, pp. 215-233, 2009.

[11] J. Hou, L. Lin, W. Zhou et al., "Identification of miRNomes in human liver and hepatocellular carcinoma reveals miR-199a/b$3 p$ as therapeutic target for hepatocellular carcinoma," Cancer Cell, vol. 19, no. 2, pp. 232-243, 2011.

[12] G. Chen, J. Wang, and Q. Cui, "Could circulating miRNAs contribute to cancer therapy?" Trends in Molecular Medicine, vol. 19, no. 2, pp. 71-73, 2013.

[13] H. Zhang, Q. Y. Li, Z. Z. Guo et al., "Serum levels of microRNAs can specifically predict liver injury of chronic hepatitis B," World Journal of Gastroenterology, vol. 18, no. 37, pp. 5188-5196, 2012.

[14] H. Zhang, Y. Guan, Y. Y. Lu et al., "Circulating miR-583 and miR-663 refer to ZHENG differentiation in chronic hepatitis B," Evidence-Based Complementary and Alternative Medicine, vol. 2013, Article ID 751341, 8 pages, 2013.

[15] Q. L. Chen, Y. Y. Lu, G. B. Zhang et al., "Progression from excessive to deficient syndromes in chronic hepatitis B: a dynamical network analysis of miRNA array data," EvidenceBased Complementary and Alternative Medicine, vol. 2013, Article ID 945245, 10 pages, 2013.
[16] H. Zhuang, "Guideline on prevention and treatment of chronic hepatitis B in China (2005)," Chinese Medical Journal, vol. 120, no. 24, pp. 2159-2173, 2007.

[17] "The standards of TCM differential syndromes of viral hepatitis," Internal Medicine Hepatopathy Committee of Chinese Traditional Medicine Association, December 1991.

[18] T. Vergoulis, I. S. Vlachos, P. Alexiou et al., "TarBase 6. 0 : capturing the exponential growth of miRNA targets with experimental support," Nucleic Acids Research, vol. 40, no. D1, pp. D222-D229, 2012.

[19] X. Wang and I. M. El Naqa, "Prediction of both conserved and nonconserved microRNA targets in animals," Bioinformatics, vol. 24, no. 3, pp. 325-332, 2008.

[20] D. W. Huang, B. T. Sherman, and R. A. Lempicki, "Systematic and integrative analysis of large gene lists using DAVID bioinformatics resources," Nature Protocols, vol. 4, no. 1, pp. 44-57, 2009.

[21] D. W. Huang, B. T. Sherman, and R. A. Lempicki, "Bioinformatics enrichment tools: paths toward the comprehensive functional analysis of large gene lists," Nucleic Acids Research, vol. 37, no. 1, pp. 1-13, 2009.

[22] S. Zheng, W. P. Tansey, S. W. Hiebert, and Z. Zhao, "Integrative network analysis identifies key genes and pathways in the progression of hepatitis $C$ virus induced hepatocellular carcinoma," BMC Medical Genomics, vol. 4, article 62, 2011.

[23] A. Krek, D. Grün, M. N. Poy et al., "Combinatorial microRNA target predictions," Nature Genetics, vol. 37, no. 5, pp. 495-500, 2005.

[24] Y. Guo, Y. Feng, N. S. Trivedi, and S. Huang, "Medusa structure of the gene regulatory network: dominance of transcription factors in cancer subtype classification," Experimental Biology and Medicine, vol. 236, no. 5, pp. 628-636, 2011.

[25] J. A. Miller, S. Horvath, and D. H. Geschwind, "Divergence of human and mouse brain transcriptome highlights Alzheimer disease pathways," Proceedings of the National Academy of Sciences of the United States of America, vol. 107, no. 28, pp. 12698-12703, 2010.

[26] D. He, Z. P. Liu, M. Honda et al., "Coexpression network analysis in chronic hepatitis B and $\mathrm{C}$ hepatic lesions reveals distinct patterns of disease progression to hepatocellular carcinoma," Journal of Molecular Cell Biology, vol. 4, no. 3, pp. 140-152, 2012.

[27] A. Spaziani, A. Alisi, D. Sanna, and C. Balsano, "Role of p38 MAPK and RNA-dependent protein kinase (PKR) in hepatitis C virus core-dependent nuclear delocalization of cyclin B1," Journal of Biological Chemistry, vol. 281, no. 16, pp. 10983-10989, 2006. 


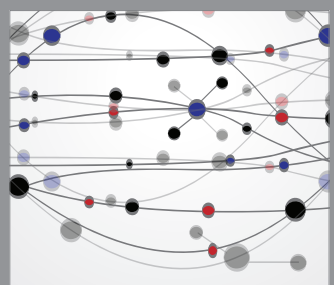

The Scientific World Journal
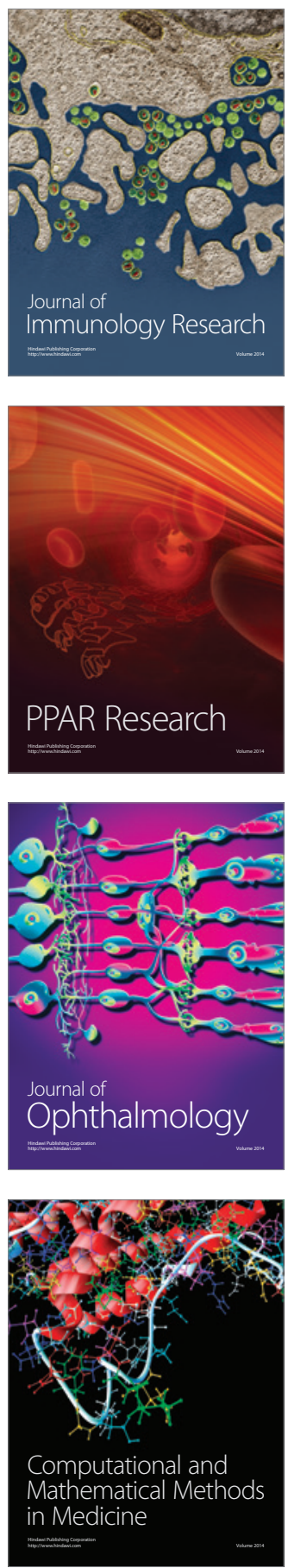

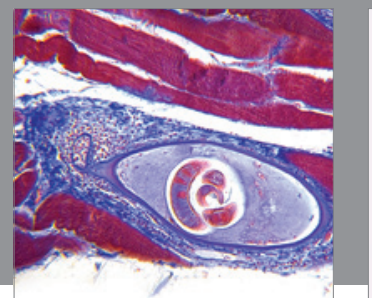

Gastroenterology

Research and Practice
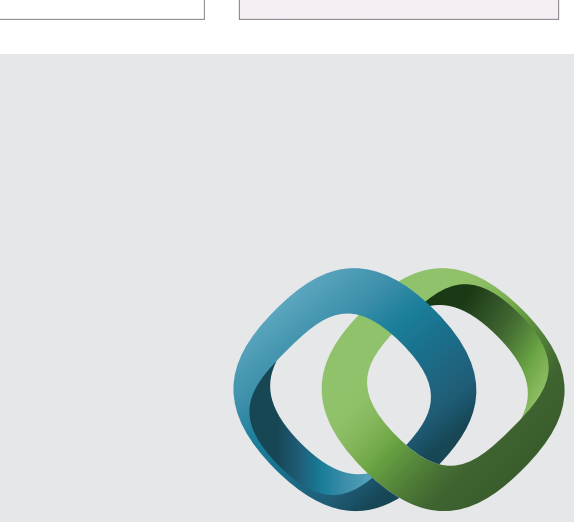

\section{Hindawi}

Submit your manuscripts at

http://www.hindawi.com
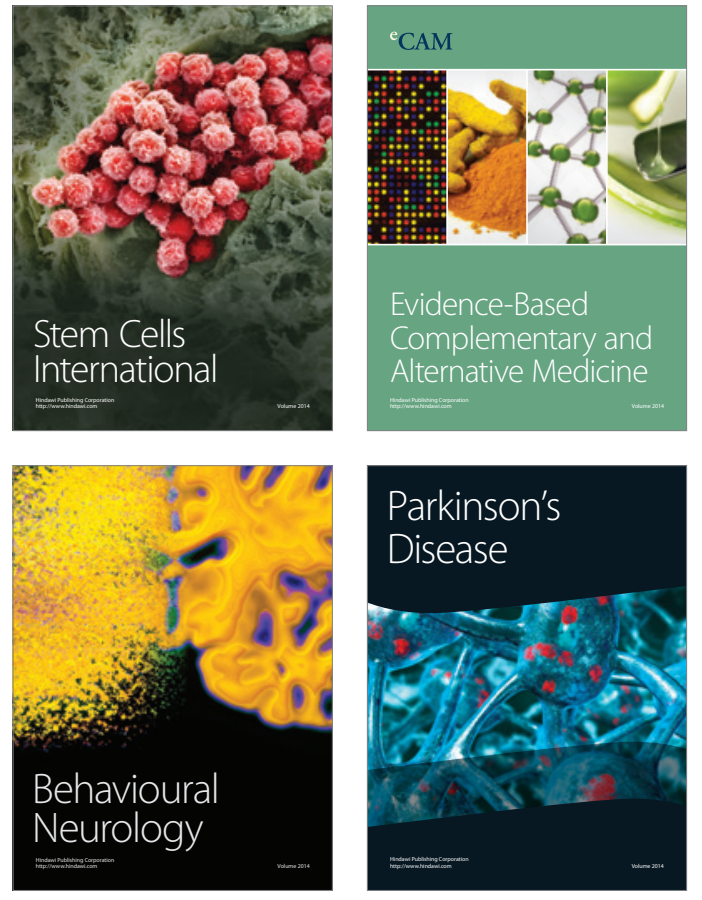
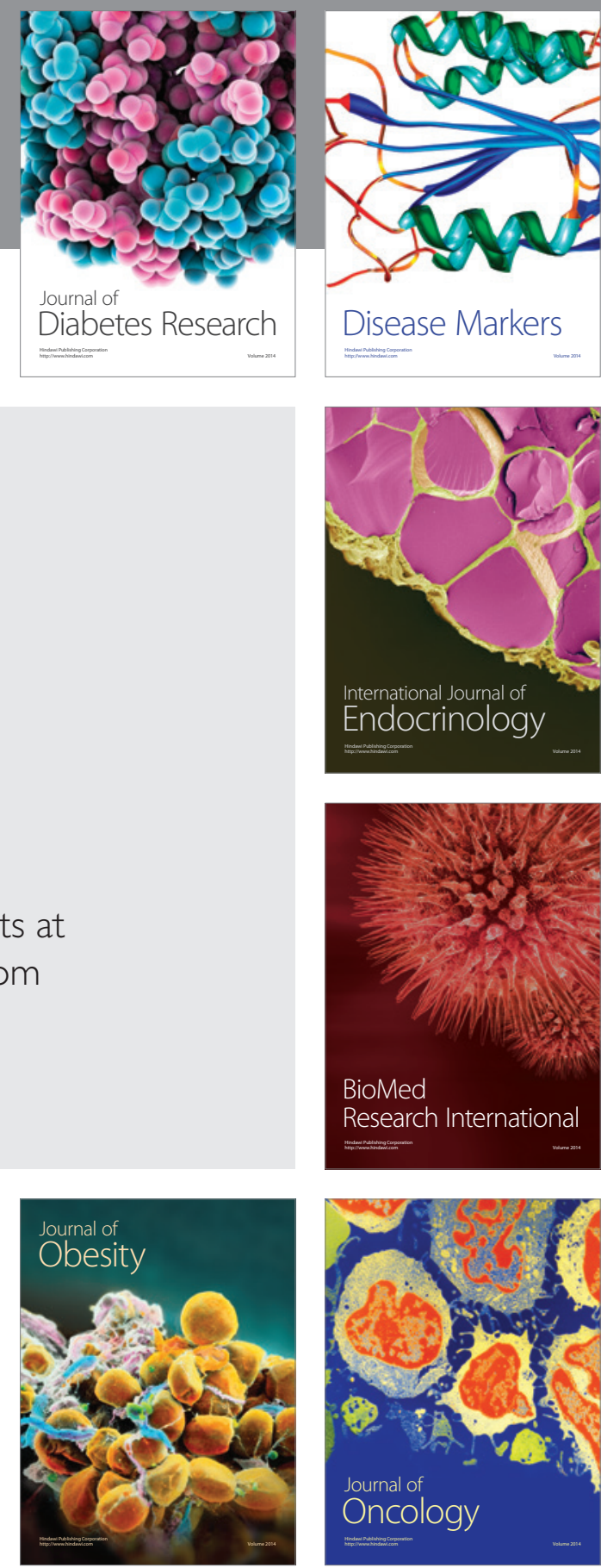

Disease Markers
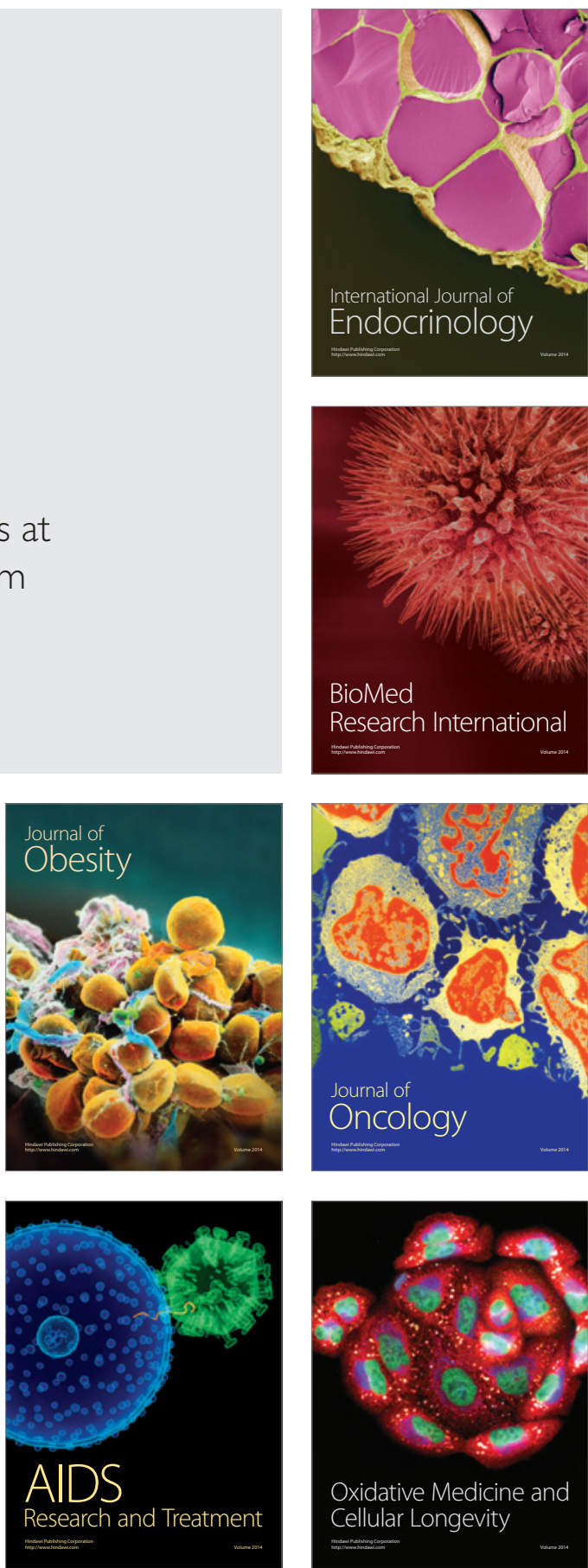